\title{
Glycosylated haemoglobin is markedly elevated in new and known diabetes patients with hyperglycaemic ketoacidosis
}

\author{
Chukwuma O Ekpebegh ${ }^{1}$, Benjamin Longo-Mbenza ${ }^{1}$, Ernesto Blanco-Blanco ${ }^{2}$
}

1. Department of Internal Medicine, Faculty of Health Sciences, Walter Sisulu University, Mthatha, Private Mail Bag X1, Mthatha, Postal code 5117, Mthatha, Eastern Cape Province, South Africa.

2. Department of Chemical Pathology, Faculty of Health Sciences, Walter Sisulu University, Mthatha, Private Mail Bag X1, Mthatha, Postal code 5117, Mthatha, Eastern Cape Province, South Africa.

\begin{abstract}
Background: Glycosylated haemoglobin (HbA1c) and random blood glucose are markers of chronic and acute hyperglycaemia respectively.

Objective: We compared HbA1c levels in ketoacidosis (DKA) occurring in known and newly diagnosed diabetes.

Methods: Retrospective review of medical records for 83 DKA admissions in 2008 and 2009 with results for HbA1c at presentation

Results: There were 52 and 31 DKA admissions in known and newly diagnosed diabetes patients respectively. Fifty of the 83 DKA admissions were in females. The mean age (per admissions) and HbA1c of all admissions are $43.4 \pm 20.3$ years $(\mathrm{n}=83)$ and $12.7 \pm 3.4 \%(\mathrm{n}=83)$ respectively. Mean HbA1c in known Type 1, known Type 2 and newly diagnosed diabetes patients were similarly very high: $12.4 \pm 3.3 \%, 12.5 \pm 3.3 \%, 13.1 \pm 3.7 \% ; \mathrm{P}=0.6828$. The HbA1c levels in newly diagnosed diabetes patients less than 30 years (likely Type 1 diabetes) and $\geq 30$ years (likely Type 2 diabetes) were similar. There was a tendency to significantly positive correlation between blood glucose and HbA1c in new diabetes patients.

Conclusions: In our setting, DKA is associated with markedly elevated HbA1c levels in known type 1, known type 2 and new onset diabetes.
\end{abstract}

Key words: Glycosylated haemoglobin, ketoacidosis, Known and newly diagnosed diabetes

DOI:http://dx.doi.org/10.4314/ahs.v14i3.5

\section{Introduction}

Glycosylated haemoglobin (HbA1c) is formed by the non-enzymatic glycation of haemoglobin and typically reflects the average blood glucose level during the life span of the red blood cell which is about 2-3 months [1]. It is currently the gold standard for assessing long term glycaemic control in diabetes mellitus [2] as it demonstrates good association with the chronic microvascular complications of diabetes such as retinopathy, nephropathy and neuropathy [3].

\section{Corresponding author: \\ Chukwuma O Ekpebegh \\ Department of Internal Medicine, \\ Faculty of Health Sciences, \\ Walter Sisulu University, Mthatha, \\ Private Mail Bag X1, \\ Postal code 5117, Mthatha, \\ Eastern Cape Province, South Africa. \\ Email: chuksekpebegh@yahoo.com \\ Telephone number: +27714516355 \\ Fax number: +27475024968}

Recently HbA1c has been included as a criterion for the diagnosis of diabetes mellitus [4]. The HbA1c level is however, of limited use in the setting of certain haemoglobinopathies, haemolytic states [5, 6]. Random blood glucose however, is a reflection of acute blood glucose level.

Diabetic ketoacodosis (DKA), a life threatening acute metabolic complication of diabetes, may occur in people previously known with diabetes or present as the first manifestation of diabetes [7]. Diabetic ketoacidosis as the first manifestation of diabetes is most often associated with Type 1 diabetes due to the rapid and marked beta cells destruction with resultant absolute insulinopenia. The presence of ketoacidosis at diagnosis of Type 1 diabetes has been shown to be a marker of depleted beta cell reserve and poor metabolic state [8]. Type 2 diabetes is however, characterized by a chronic asymptomatic pre-clinical phase which progresses through a period of insulin independence to insulin dependence. Diabetic ketoacidosis which depicts a state of severe insulin deficiency is increasingly recognized as the initial manifestation of Type 2 diabetes [9].

The expectation is that of a lower HbA1c in Type 1 diabetes patients with DKA as the first manifestation of 
diabetes when compared with Type 2 diabetes patients with DKA as the first manifestation of diabetes. This is because Type 2 diabetes is characterized by a longer period of asymptomatic hyperglycaemia than Type 1 diabetes. We assessed and compared HbA1c (marker of chronic hyperglycaemia) and random plasma glucose levels (marker of acute hyperglycaemia) at presentation with DKA in patients with Known diabetes (Types 1 and 2) and newly diagnosed diabetes.

\section{Materials and methods}

Subjects: These were 83 admissions for DKA during the period 2008 and 2009 for which HbA1c results were available. Repeat admissions for DKA in patients that were already known to have diabetes before the year 2008 were treated as separate admissions. No patient who was admitted during the period of 2008 and 2009 with DKA as the initial manifestation of diabetes represented to us with DKA during the 2 year period.

Study design: Retrospective review of medical records over the 2-year period of 2008 and 2009. Known Types 1 and 2 diabetes were as categorized in the case records.

Ethical approval: This was obtained from the Ethics committee, Faculty of Health Sciences, Walter Sisulu University, Eastern Cape Province of South Africa.

Glycosylated haemoglobin assay: This was done by the National Health Laboratory Services (NHLS), Mthatha using Roche/Hitachi COBAS 6000 analyzer (Cobas c 501 module) with A1C-2 diagnostic system supplied by Roche (Roche Diagnostics GmbH, Mannheim, Germany). The assay was a turbidimetric inhibition immunoassay.
Plasma glucose assay: This was determined by an enzymatic reference method using hexokinase on the Roche/Hitachi (Cobas c 501 module), (Roche Diagnostics GmbH, Mannheim, Germany).

Statistical analysis: Qualitative data were expressed as frequency and proportions (\%). Continuous data were reported as mean $\pm \mathrm{SD}$. In univariate analysis, the means of the three study groups (Known Type 1, Known Type 2, Newly diagnosed diabetes) were compared using oneway Anova with Bonferroni Post-Hoc test. Chi-Square test was used to compare proportions. The Pearson simple correlation coefficient $r$ and determination coefficient $\mathrm{R}^{2}$ from the line regression equation served to examine the association between blood glucose and HbA1c. Statistical package was SPSS (version 17, Chicago, Illinois). $\mathrm{P} \leq 0.05$ defined statistical significance between groups.

\section{Results}

There were 83 admissions comprising 31 that presented with the index DKA as the first manifestation of diabetes and 52 in patients already known with diabetes. Known Types 1 and 2 diabetes accounted for 20 and 32 admissions respectively in known diabetic patients. Females were $60.2 \%(n=50 / 83)$ of all admissions. The mean age per admissions was $43.4 \pm 20.3$ years $(n=83)$. Mean blood glucose and HbA1c levels were $33.5 \pm 14.9$ $\mathrm{mmol} / \mathrm{L}(\mathrm{n}=83)$ and $12.7 \pm 3.4 \%(\mathrm{n}=83)$ respectively. The proportions of admissions in various $\mathrm{HbA} 1 \mathrm{c}$ categories were $1.2 \%(\mathrm{n}=1 / 83)$ for HbA1c $<7 \%, 20.5$ $\%(n=17 / 83)$ for HbA1c of 7-9.9\%, 55.4\% ( $n=46 / 83)$ for $\mathrm{HbA1c}$ of $10-15 \%$ and $22.9 \%(n=19 / 83)$ for $\mathrm{HbA} 1 \mathrm{c}>15 \%$.

Table 1: Demographic and clinical parameters of DKA admissions according to diabetes type

\begin{tabular}{lllll}
\hline & $\begin{array}{l}\text { Known } \\
\text { Type 1 DM }\end{array}$ & $\begin{array}{l}\text { Known } \\
\text { Type 2 DM }\end{array}$ & New DM & P value \\
\hline Female $(\%)$ & $70(14 / 20)$ & $56.3(18 / 32)$ & $58.1(18 / 31)$ & 0.59 \\
Age $<30$ years $(\%)$ & $85(17 / 20)$ & $3.1(1 / 32)$ & $35.5(11 / 31)$ & $<0.01$ \\
Hyperosmolar $(\%)$ & $5(1 / 20)$ & $15.6(5 / 32)$ & $32.3(10 / 31)$ & 0.04 \\
$\begin{array}{l}\text { Blood glucose }>33.3 \\
\text { mmol/L }(\%)\end{array}$ & $40(8 / 20)$ & $28.1(9 / 32)$ & $41.9(13 / 31)$ & 0.48 \\
$\begin{array}{l}\text { Serum urea }>7 \mathrm{mmol} / \mathrm{L} \\
(\%)\end{array}$ & $70(14 / 20)$ & $18.8(6 / 32)$ & $41.9(13 / 31)$ & $<0.01$ \\
$\begin{array}{l}\text { Haemoglobin }<10 \mathrm{~g} / \mathrm{L} \\
(\%)\end{array}$ & $15(3 / 20)$ & $28.1(7 / 31)$ & $10(3 / 30)$ & 0.40 \\
\hline
\end{tabular}


Table 1 shows that patients with known Type 1 in contrast to known Type 2 diabetes were predominantly below 30 years. Hyperosmolality (calculated effective serum osmolality $>320 \mathrm{mosm} / \mathrm{Kg}$ ) was more common in newly diagnosed diabetes than known Types 1 and 2 diabetes patients. The proportion of admissions with elevated serum urea (serum urea $>7 \mathrm{mmol} / \mathrm{L}$ ) was higher with known Type 1 than other groups.

Table 2: Mean HbA1c and proportions of admissions categorized by HbA1c and diabetes type

\begin{tabular}{lllll}
\hline & $\begin{array}{l}\text { Known } \\
\text { Type 1 DM }\end{array}$ & $\begin{array}{l}\text { Known } \\
\text { Type 2 DM }\end{array}$ & New DM & P value \\
\hline Mean HbA1c $(\%)$ & $12.4+3.3$ & $12.5+3.3$ & $13.1+3.7$ & 0.68 \\
HbA1c Categories & & & & 0.87 \\
& & & & \\
$<7 \%$ & $5(1 / 20)$ & $0(0 / 32)$ & $0(0 / 31)$ & \\
$7-9.9 \%$ & $25(5 / 20)$ & $21.9(7 / 32)$ & $16.1(5 / 31)$ & \\
$10-15 \%$ & $50(10 / 20)$ & $53.1(17 / 32)$ & $61.3(19 / 31)$ & \\
$>15 \%$ & $25(5 / 20)$ & $21.9(7 / 32)$ & $22.6(7 / 31)$ & \\
\hline
\end{tabular}

Table 2 shows comparably high mean HbA1c levels irrespective of diabetes type. The majority of admissions had HbA1c in the range of $10-15 \%$ in all diabetes types. The only admission with an $\mathrm{HbA1c}$ of less than $7 \%$ which is the American Diabetes Association (ADA) criterion for optimal glycaemic control [10] was a known Type 1 diabetes patient with infection as the precipitating cause for DKA.

Table 3: Proportions of admissions categorized by HbA1c and precipitating factor

\begin{tabular}{lllllll}
\hline & Infection & CVD & None Compliance & None & Others & P value \\
\hline$<7 \%$ & $5.6(1 / 18)$ & $0(0 / 2)$ & $0(0 / 9)$ & $0(0 / 50)$ & $0(0 / 4)$ & \\
$7-9.9 \%$ & $16.7(3 / 18)$ & $50(1 / 2)$ & $11.1(1 / 9)$ & $22(11 / 50)$ & $25(1 / 4)$ & 0.45 \\
$10-15 \%$ & $55.6(10 / 18)$ & $50(1 / 2)$ & $33.3(3 / 9)$ & $58(29 / 50)$ & $75(3 / 4)$ & \\
$>15 \%$ & $22.2(4 / 18)$ & $0(0 / 2)$ & $55.6(5 / 9)$ & $20(10 / 50)$ & $0(0 / 4)$ & \\
\hline
\end{tabular}

CVD: Cerebrovascular disease.

Table 3 shows high HbA1c levels irrespective of the precipitating factor for DKA. The majority of admissions associated with non compliance as the precipitating factor had HbA1c levels of $\geq 10 \%$.

Table 4: Proportions of admissions in newly diagnosed diabetes categorized by HbA1c and age 


\begin{tabular}{llll}
\hline & $\begin{array}{l}\text { Age }<30 \text { years } \\
(\text { Likely Type 1 DM) }\end{array}$ & $\begin{array}{l}\text { Age } \geq 30 \text { years } \\
\text { (Likely Type 2 DM) }\end{array}$ & P value \\
\hline$<7 \%$ & $0(0 / 11)$ & $0(0 / 20)$ & 0.19 \\
$7-9.9 \%$ & $0(0 / 11)$ & $25(5 / 20)$ & \\
$10-15 \%$ & $72.7(8 / 11)$ & $55(11 / 20)$ & \\
$>15 \%$ & $27.3(3 / 11)$ & $20(4 / 20)$ & \\
\hline
\end{tabular}

Table 4 shows the proportions of admissions in newly diagnosed diabetes categorized by HbA1c and age. The 11 newly diagnosed diabetes patients who were less than 30 years of age and therefore, likely to be predominantly Type 1 diabetes all had HbA1c levels $\geq 10 \%$. These 11 patients who were below 30 years of age had similar HbA1c profiles as patients $\geq 30$ years who are likely to be mainly Type 2 diabetics.

Figures 1 to 3 show linear regression lines interposed on scatter plots of blood glucose and HbA1c for, known Type 2 diabetes (Figure 1), known Type 1 diabetes (Figure 2) and newly diagnosed diabetes patients (Figure 3). There was a tendency to significant positive correlation between blood glucose and Hba1c in newly diagnosed diabetes $(n=31), r=0.346 ; P=0.056$. There was non-significant correlations between blood glucose and HbA1 $\mathrm{c}$ in Type 2 diabetes $(\mathrm{n}=32), \mathrm{r}=0.156 ; \mathrm{P}=0.394$ and Type 1 diabetes $(n=20), r=-0.076 ; P=0.076$.

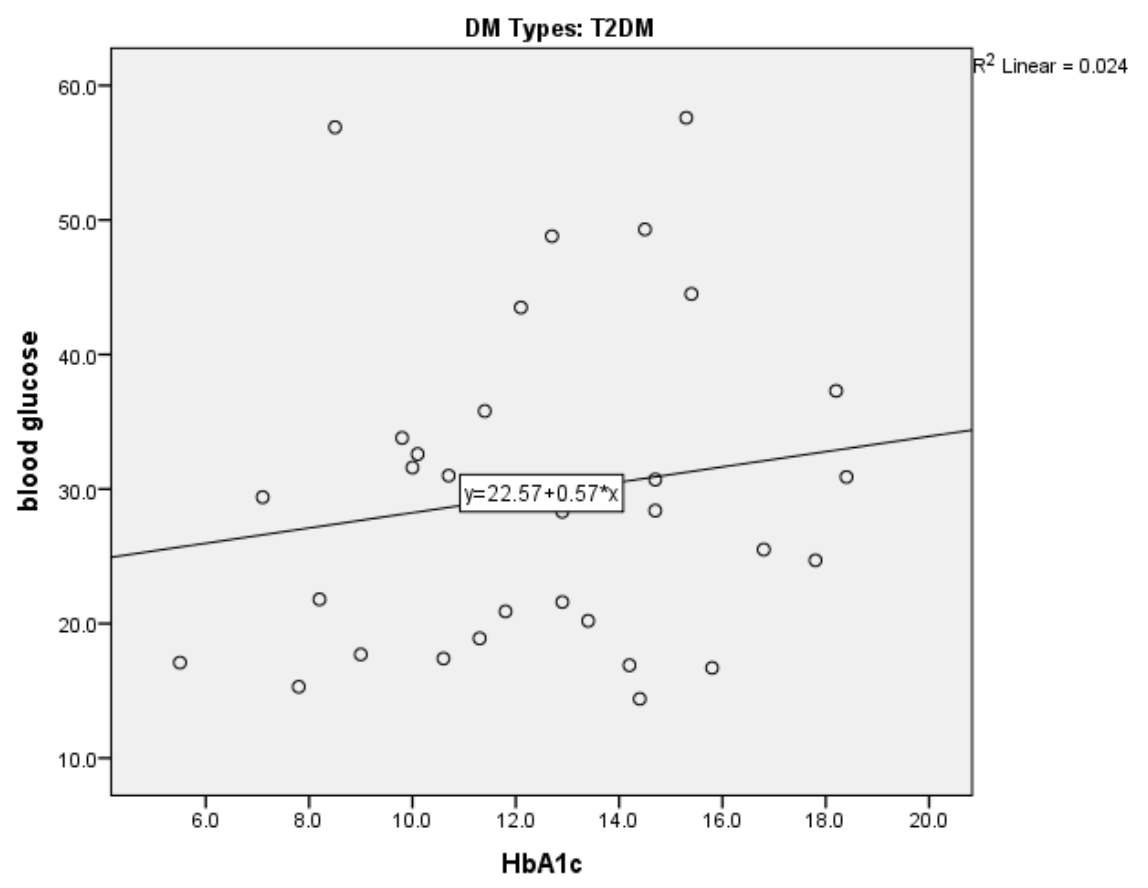

Figure 1. Linear regression equation of blood glucose and HbA1c in Type 2 diabetes patients. 


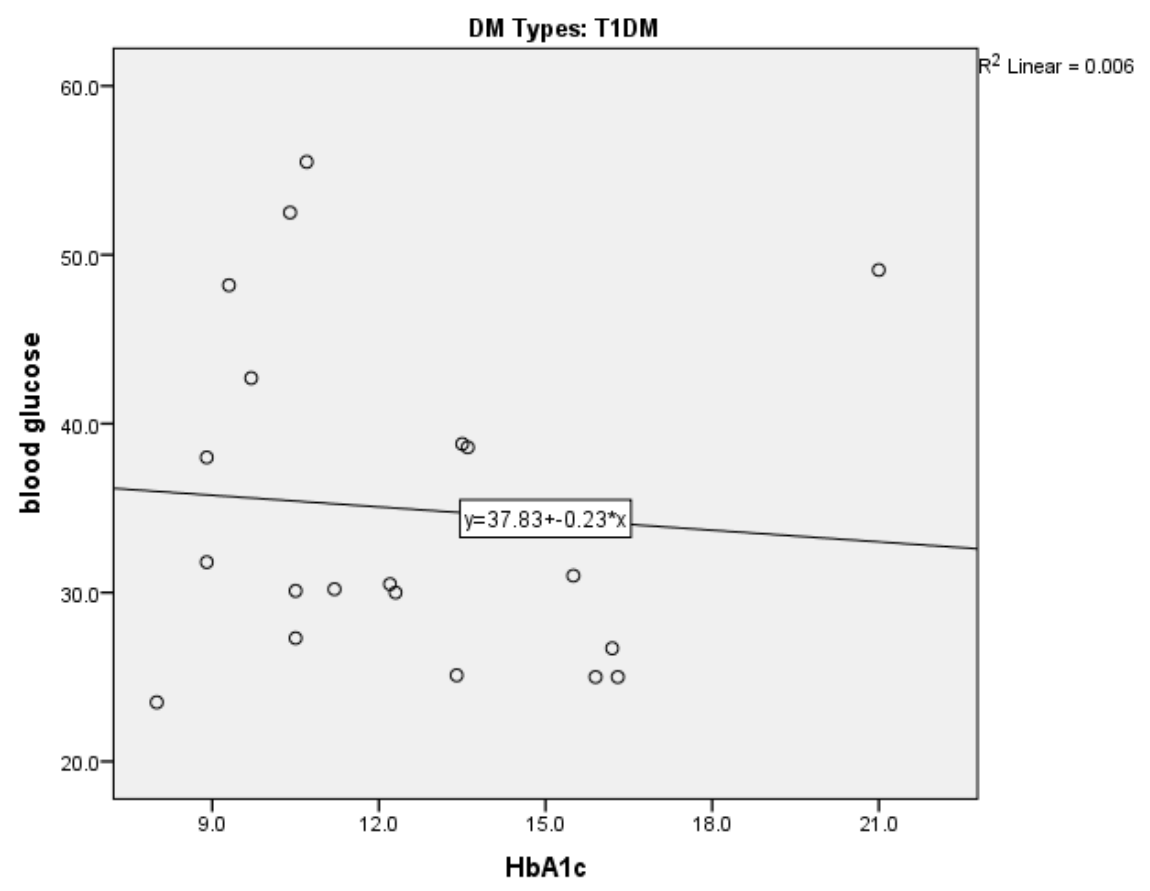

Figure 2. Linear regression equation of blood glucose and HbA1c in Type 1 diabetes patients.

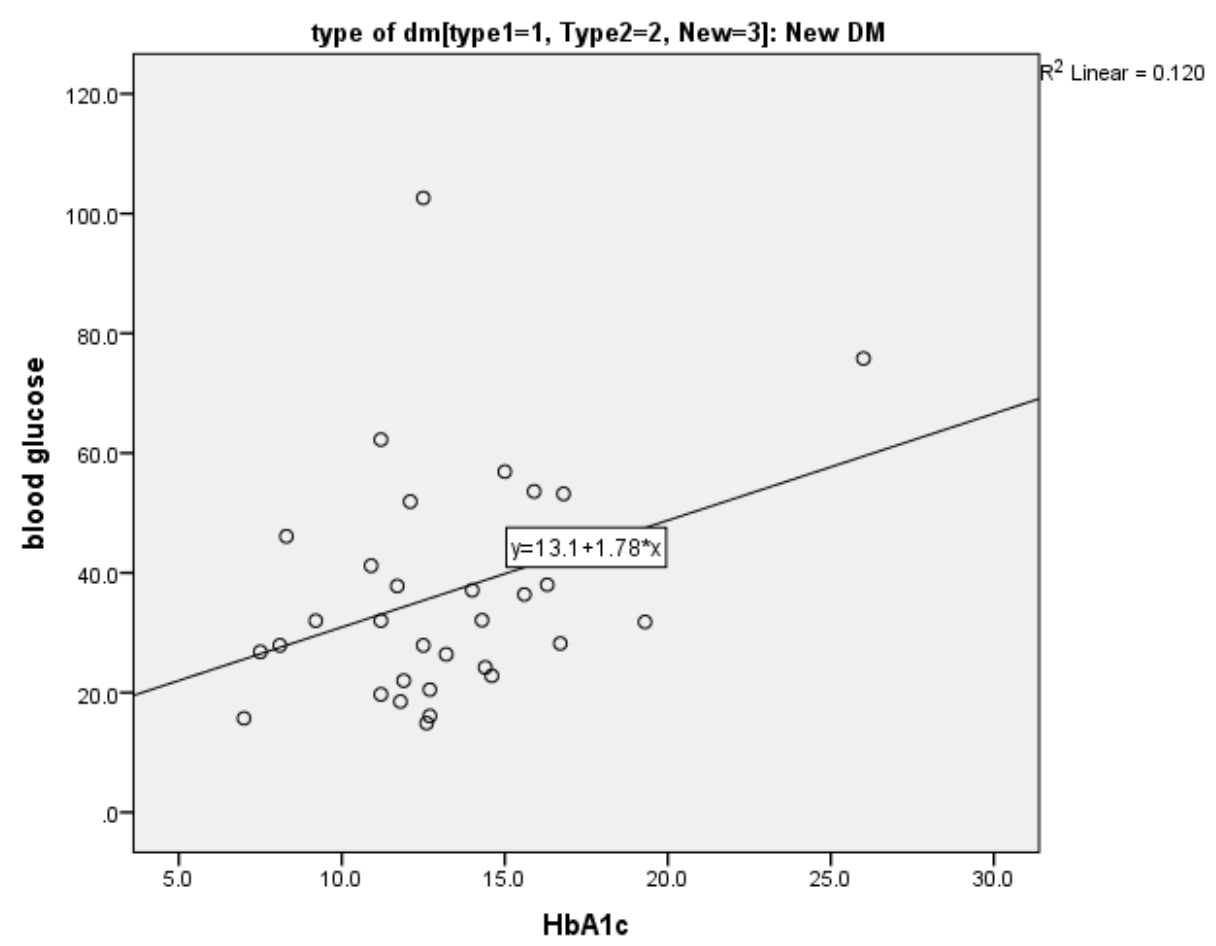

Figure 3. Linear regression equation of blood glucose and HbA1c in newly diagnosed diabetes patients. 


\section{Discussion}

The major finding of this retrospective review is that almost all admissions for DKA were associated with markedly elevated HbA1c levels at presentation. This was regardless of whether patients were already known with Types 1 and 2 diabetes or only just diagnosed with diabetes at presentation with the index DKA. Although DKA is regarded as an acute metabolic complication of diabetes, the findings of very high $\mathrm{HbA1c}$ levels in this study, suggests that in our setting, DKA occurs in the background of chronic hyperglycaemia in both known and newly diagnosed diabetes. We have therefore since stopped requesting for $\mathrm{HbA1c}$ at presentation with DKA. We rather perform HbA1c during clinic visit at 3 months following discharge from a DKA related admission.

The tendency $(\mathrm{P}=0.06)$ to significant positive correlation between blood glucose and HbA1c levels in newly diagnosed diabetes is likely a reflection of concurrent increase in both blood glucose and HbA1c with no interference from glucose lowering therapy. The negative correlation that was seen with Type 1 diabetes, though not significant may be partly explained by sudden onset severe hyperglycaemia due to marked insulinopenia such that the blood glucose levels become discordantly higher than the HbA1c levels. The rather gradual slope seen with Type 2 diabetes in contrast to the steep slope of newly diagnosed diabetes group is possibly an effect of glucose lowering therapy.

Our findings are similar to that of another study [11] which reported HbA1c levels of $10.4 \%$ - 16.9\% in patients with DKA as the first manifestation of diabetes. One might have expected the newly diagnosed diabetes patients less than 30 years to have lower HbA1c levels than patients who are 30 years of age and above. This is because the majority of newly diagnosed diabetes patients who are less than 30 years are likely to have Type 1 diabetes [12] which is expected to have an acute pathogenesis. A study [12] of 60 persons with diabetes all less than 30 years of age who were classified as Types $1 \mathrm{~A}, 1 \mathrm{~B}$ and Type 2 based on beta cell autoimmunity and insulin reserve showed that the proportions of Types $1 \mathrm{~A}, 1 \mathrm{~B}$ and 2 were $55 \%, 30 \%$ and $15 \%$ respectively. The high HbA1c levels in our newly diagnosed diabetes patients below 30 years indicates that even where DKA is the first manifestation of 'Type 1 diabetes, there has been hyperglycaemia of sufficiently long duration of time to result in markedly elevated HbA1c levels. The Diabetes Prevention Trial-Type 1 (DPT) found a relatively normal mean $\mathrm{HbA} 1 \mathrm{c}$ of $6.41 \pm 1.5 \%$ at diagnosis, in asymptomatic Type 1 diabetes [13], suggesting that DKA may be a late manifestation of Type 1 diabetes. Although screening for Type 1 diabetes is currently not the norm, early presentation and treatment is desirable before glycaemia deteriorates to the degree where DKA supervenes.

The study showed similar HbA1c profiles irrespective of the precipitating factor (Table 3). The majority of admissions that were associated with non-compliance to medications as the precipitating factor had markedly elevated $\mathrm{HbA} 1 \mathrm{c}$ levels indicating that these are persons with long standing poor glycaemic control which probably rendered them easily susceptible to developing ketoacidosis.

The association between elevated HbA1c and DKA in new onset and known diabetic patients may be related to chronic hyperglycaemia as this has been hypothesized to cause metabolic decompensation by impairing the functions of the beta cell reserve and insulin receptor via the mechanism of glucose toxicity [14]. Perhaps, improving long term glycaemic control in the known diabetic patient may reduce presentations for DKA.

The limitations of this study include categorizing patients with DKA in new onset diabetes with the age cut off of 30 years. There was no data on markers of beta cell autoimmunity and insulin reserve which will have allowed for better classification as Type 1 or 2 diabetes. However, a recent study [15] in our centre showed the majority of patients below 30 years of age with DKA at diagnosis of diabetes to be Type 1 based on positive anti-glutamic acid decarboxylase status and low plasma C-peptide response to intravenous injection of glucagon. The presence of Type 2 diabetes cannot be excluded particularly in young persons with $\mathrm{HbA1c}$ above $10 \%$ considering the increasing prevalence of Type 2 diabetes in the young [16].

\section{Conclusion}

Diabetic ketoacidosis in our environment is associated with very high HbA1c levels irrespective of the Type and duration of diabetes or precipitating factor for DKA. The clinical care implications are that stable patients with very high HbA1c levels should have glycaemia aggressively managed to avert the occurrence of DKA. There is no value in measuring HbA1c levels at presentation with DKA.

\section{References}

1. Cohen RM, Franco RS, Khera PK, Smith EP, Lindsell CJ and Ciraolo PJ et al. Red cell life span heterogeneity 
in haematologically normal people is sufficient to alter HbA1c. Blood 2008; 112: 4284-4291.

2. Toh MP, Heng BH, Sum CF, Jong M, Chionh SB and Cheah, JT. Measuring the quality of care of diabetic patients at the specialist outpatient clinics in public hospitals in Singapore. Ann Acad Med Singapore 2007; 36. $980-986$.

3. Stratton IM, Adler AI, Neil HA, Mathews DR, Manley SE and Cull CA et al. Association of glycaemia with macrovascular and microvascular complications of type 2 diabetes (UKPDS 35): prospective observational study. BMJ 2000; 321: 405-412.

4. International Expert Committee. International Expert Committee report on the role of A1c assay in the diagnosis of diabetes: Diabetes Care 2009; 32: $1327-$ 1334.

5. Bhat VS, Dewan KK, Krishnaswamy PR. Diagnostic dilemma of $\mathrm{HbA1c}$ detection in presence of a haemoglobinopathy: A case report: Indian J Clin Biochem 2011: 26. 91-95.

6. Kutter D, Thoma J. Hereditary spherocytosis and other haemolytic anomalies distort diabetic control by glycated haemoglobin. Clin Lab 2006; 52: 477-481.

7. Newton CA, Raskin P. Diabetic ketoacidosis in Type 1 and type 2 diabetes mellitus. Clinical and Biochemical differences: Arch Intern Med 2004; 164: 1925-1931.

8. Fernandez CM, Montana E, Camps I, Biarnes J, Merino JF and Escriba JM et al. Ketoacidosis at diagnosis is predictive of lower residual beta cell function and poor metabolic control in type 1 diabetes. Diabetes Metab 1996; 22: 349-355.

9. Umpierrez GE, Woo W, Hagopian WA, Issacs SD, Palmer JP and Gaur LK et al. Immunogenetic analysis suggests different pathogenesis for obese and lean African-Americans with diabetic ketoacidosis. Diabetes Care 1999, 22.1517-1523.

10. American Diabetes Association. Standards of medical care in diabetes. Diabetes Care 2012; 35 (Suppll1): S11-S63.

11. Henderson DC, Cagliero E, Copeland PM, Louie PM, Borba CP and Fan X et al. Elevated haemoglobin A1c as a possible indication of diabetes mellitus and diabetic ketoacidosis in Schizophrenia patients receiving atypical anti-psychotics. J Clin Psychiatry 2007; 68: 533541.

12. Ekpebegh CO, Longo-Mbenza B. Clinical, immunologic and insulin secretory characteristics of young black South African patients with diabetes: Hospital based single centre study. Diabetes Res Clin Pract 2013; 99: 380-384.

13. Triolo TM, Barker JM and Chase HP. (The DPT-1 study group). Diabetic subjects diagnosed through the Diabetes Prevention Trial-Type 1 (DPT-1) are often asymptomatic with normal A1c at diabetes onset. Diabetes Care 2009; 32: 769-773.

14. Mauvais-Jarvis F, Sobongwi E, Porcher R, Riveline J, Kevorkian J and Vaisse C. Ketosis prone type 2 diabetes in patients of sub-Saharan origin: Diabetes 2004; 53: 645-653.

15. Ekpebegh C, Longo-Mbenza B, Blanco-Blanco E. Islet immunity and beta cell reserve of indigenous black South Africans with ketoacidosis at initial diagnosis of diabetes. Ethn Dis 2013; 23: 196-201.

16. Bloomgarden ZT. Type 2 diabetes in the young. Diabetes Care 2004; 27: 998-1010. 